\title{
Accurate electronic band gap of pure and functionalized graphane from GW calculations
}

\author{
S. Lebègue, ${ }^{1}$ M. Klintenberg, ${ }^{2}$ O. Eriksson, ${ }^{2}$ and M. I. Katsnelson ${ }^{3}$ \\ 1 Laboratoire de Cristallographie, Résonance Magnétique et Modélisations (CRM2, \\ UMR CNRS 7036) Institut Jean Barriol, Nancy Université BP 239, \\ Boulevard des Aiguillettes 54506 Vandoeuvre-lès-Nancy,France \\ ${ }^{2}$ Department of Physics and Materials Science, Uppsala University, Box 530, SE-75121, Uppsala, Sweden \\ 3 Institute for Molecules and Materials, Radboud University Nijmegen, \\ Heyendaalseweg 135, NL-6525 AJ, Nijmegen, The Netherlands
}

(Dated: July 1, 2021)

\begin{abstract}
Using the GW approximation, we study the electronic structure of the recently synthesized hydrogenated graphene, named graphane. For both conformations, the minimum band gap is found to be direct at the $\Gamma$ point, and it has a value of $5.4 \mathrm{eV}$ in the stable chair conformation, where $\mathrm{H}$ atoms attach $\mathrm{C}$ atoms alternatively on opposite sides of the two dimensional carbon network. In the meta-stable boat conformation the energy gap is $4.9 \mathrm{eV}$. Then, using a supercell approach, the electronic structure of graphane was modified by introducing either an hydroxyl group or an $\mathrm{H}$ vacancy. In this last case, an impurity state appears at about $2 \mathrm{eV}$ above the valence band maximum.

PACS numbers: 81.05.Uw, 71.15.Mb, 71.10.-w
\end{abstract}

After the discovery of graphene 1 and of its extraordinary electronic properties [2, 3, 4] the chemical functionalization of graphene has become the focus of special interest in contemporary materials science. Being first a truly two-dimensional crystal and demonstrating high electron mobility [1], graphene is ideal for modern electronics which is essentially two-dimensional 2]. At the same time, the peculiar gapless "ultrarelativistic" energy spectrum of graphene [3, 4] makes the creation of a "carbon transistor" based on $p-n$ junctions highly nontrivial, due to the anomalous transparency ("Klein tunneling" [5]). Nanoscale graphene single-electron transistor has been demonstrated already [6] but it has a relatively restricted domain of potential applications. Therefore, to transform graphene into a semiconductor with a conventional electron spectrum keeping its two-dimensionality is a real challenge in the field, and chemical functionalization is considered as one of the most promising ways to solve the problem.

A new derivative of graphene was recently synthesized[7], where hydrogenation turned graphene into what now is called graphane. Previously this compound was studied theoretically, and it is actually an excellent example of a new and interesting material which was predicted from first principles theory [8, 9] before it was synthesised experimentally. In the calculations of the electronic structure it was found that graphane is a semiconductor with a rather wide energy gap [8, 9]. In the experiment [7] the adsorption of hydrogen on graphene was indeed observed to result in that a gap opened up in the electron states. Hence the adsorption of hydrogen turned the highly conductive graphene into insulating graphane, in accordance with the theoretical predictions. [8, 9] However, the exact value of the band gap is still experimentally unknown. Previous theoretical calculations giving values from 3.5 $\mathrm{eV}[8]$ to $3.8 \mathrm{eV}[10]$ use a standard density functional with generalized gradient approximation (GGA). It is well known that, in general, this approach is not reliable to calculate energy gaps in semiconductors often giving inaccurate results (the so called "gap problem" [11]). At the same time, knowledge about the size of this gap is crucial in order to assess the possibility of using graphane in electronics applications and for use in devices.

The reason for a band gap opening up when hydrogen is adsorbed on graphene is that $\mathrm{sp}^{2}$ bonded $\mathrm{C}$ atoms become $\mathrm{sp}^{3}$ bonded atoms, where three of the four covalent $\mathrm{sp}^{3}$ bonds are saturated by $\mathrm{C}$ atoms and the fourth covalent bond is saturated by an $\mathrm{H}$ atom. This is for instance illustrated in the theoretical part of Ref.7 and is also known from the adsorption of single hydrogen atoms on graphene 12]. In accordance to expectations this change in chemical binding is also reflected in the electronic structure, where $\mathrm{sp}^{3}$ bonded $\mathrm{C}$ atoms do not display a so called $\pi^{*}$ peak in the x-ray absorption spectrum[12]. Hence the basic reason for the drastic change in conductivity is in accordance to expectations for $\mathrm{sp}^{3}$ bonded carbon (i.e. diamond), with an insulating behaviour. There is of course no reason to expect that the size of the band gap of graphane should be similar to that of diamond, even though there are similarities in the nature of the chemical binding. Hence measurements or accurate theoretical calculations are needed, and in this article we present a calculation based on the GW approximation.

Due to the well-known deficiencies of DFT [13] to treat excited states, (for example the band gaps in the LDA or GGA approximations are much smaller than the ex- 
perimental values), we have used the GW approximation (GWA) of Hedin 14, 15] to study the electronic structure of graphane. In this formalism, the Kohn-Sham equations [13 are replaced by the quasiparticle equation: $\left(T+V_{e x t}+V_{h}\right) \psi_{\mathbf{k} n}(\mathbf{r})+\int d^{3} r^{\prime} \Sigma\left(\mathbf{r}, \mathbf{r}^{\prime}, E_{n}(\mathbf{k})\right) \psi_{\mathbf{k} n}\left(\mathbf{r}^{\prime}\right)$

$$
=E_{n}(\mathbf{k}) \psi_{\mathbf{k} n}(\mathbf{r})
$$

where $T$ is the free-electron kinetic energy operator, $V_{e x t}$ the external potential due to the ion cores, $V_{h}$ the Hartree potential, $\Sigma$ the self-energy operator, and $E_{n}(\mathbf{k})$ and $\psi_{\mathbf{k} n}(\mathbf{r})$ are respectively the quasiparticle energy and wave function.

An adequate approximation for the self-energy operator is to write it as the product of the Green's function and the screened Coulomb interaction $W$, which yields to the so-called GW approximation.

$$
\Sigma\left(\mathbf{r}, \mathbf{r}^{\prime}, \omega\right)=\frac{i}{2 \pi} \int d \omega^{\prime} G\left(\mathbf{r}, \mathbf{r}^{\prime}, \omega+\omega^{\prime}\right) e^{i \delta \omega^{\prime}} W\left(\mathbf{r}, \mathbf{r}^{\prime}, \omega^{\prime}\right)
$$

Then, since the difference $\hat{\Sigma}-\hat{V}_{x c}$ between the self-energy and the Kohn-Sham exchange and correlation potential is small, a perturbation theory is used to write the QP Hamiltonian:

$$
\hat{H}^{q p}=\hat{H}_{K S}+\left(\hat{\Sigma}-\hat{V}_{x c}\right)
$$

Finally, the QP energies are obtained by expanding the real part of self-energy to first order around $\epsilon_{n}^{D F T}(\mathbf{k})$ and assuming that the the QP wave function $\psi_{\mathbf{k} n}$ and KohnSham wave function $\Psi_{\mathbf{k} n}$ are identical:

$\left.\operatorname{Re} E_{n}(\mathbf{k})\right)=\epsilon_{n}^{D F T}(\mathbf{k})+Z_{n \mathbf{k}} \times$

$\left[\left\langle\Psi_{\mathbf{k} n}^{D F T}\left|\operatorname{Re} \Sigma\left(\mathbf{r}, \mathbf{r}^{\prime}, \epsilon_{n}(\mathbf{k})\right)\right| \Psi_{\mathbf{k} n}^{D F T}\right\rangle-\left\langle\Psi_{\mathbf{k} n}^{D F T}\left|V_{x c}^{D F T}(r)\right| \Psi_{\mathbf{k} n}^{D F T}\right\rangle\right.$

where the QP renormalization factor $Z_{n \mathbf{k}}$ is given by

$$
Z_{n \mathbf{k}}^{D F T}=\left[1-\left\langle\Psi_{\mathbf{k} n}^{D F T}\left|\frac{\partial}{\partial \omega} \operatorname{Re} \Sigma\left(\mathbf{r}, \mathbf{r}^{\prime}, \epsilon_{n}(\mathbf{k})\right)\right| \Psi_{\mathbf{k} n}^{D F T}\right\rangle\right]^{-1} .
$$

Therefore, it appears that the DFT eigenvalues $\epsilon_{n}(\mathbf{k})$ are corrected by the GW approximation, giving a practical scheme to compute reliable excited state properties 16, 17, 18.

Here we have used the code VASP 19] (Vienna Abinitio simulation package), implementing the projector augmented waves (PAW) method[20] to compute the ground state and excited state properties of graphane. First, we obtained a reliable geometry of the structure by optimizing it for the two possible conformations (see below), using the generalized gradient approximation 21]. Contrary to Sofo et al. [8], we have chosen to simulate completely isolated sheets of graphane, although the resulting bandstructure is very close to that other [8]. For all the calculations, we have used the default cut-off for the wavefunction. During the optimization of the structures, a k-point grid 22] of $10 \times 10 \times 1$ was used. Then, using the relaxed geometries, a final run was performed with a $16 \times 16 \times 1$ grid. Finally, these ground state calculations were used to compute the quasiparticle bandstructure with VASP, following the method described in Ref. 23. Two hundred bands were used for the summation over bands in the calculation of the polarisability and the self-energy, and a cut-off of $150 \mathrm{eV}$ was used for the size of the polarisability matrices. For details about the implementation of the GW approximation within the PAW formalism, see also Refs. 24, 25. Notice that the GW approximation was applied successfully to study the properties of graphene nanoribbons [26, 27], of graphene 28], and of carbon nanotubes [29].

Graphane is made of a hexagonal network of carbon atoms, with the orbitals being of the $\mathrm{sp}^{3}$ kind and bonded to hydrogen atoms, with a carbon/hydrogen ratio of one. It exists in two conformers [8], the chair-like conformer and the boat like conformer. In the chair conformer, all $\mathrm{C}-\mathrm{C}$ bonds are equivalent and the hydrogen atoms are bonded alternatively on each side of the carbon plane (see Fig.1 of Ref:8). The boat conformer has two types of C-C bonds, depending on whether the carbons atoms are bonded to hydrogen atoms which are on the same side of the carbon plane or not. Our calculations indicate that the chair-like conformer is favored by about $0.051 \mathrm{eV} /$ atom, in very good agreement with the value of Sofo et al.[8], who found $0.055 \mathrm{eV} /$ atom. Also, for the chair-like conformer the bond lengths are $1.53 \AA$ (for the $\mathrm{C}-\mathrm{C}$ bonds) and $1.11 \AA$ (for the $\mathrm{C}-\mathrm{H}$ bonds), while for the boat-like conformer, the C-C bonds are $1.57 \AA$ and $1.54 \AA$ long, and the $\mathrm{C}-\mathrm{H}$ bonds are $1.10 \AA$ long. These values are essentialy identical to the one of Ref. 8. In Fig1 we show the partial density of states (pDOS) for ] the stable chair conformer, and it is clear that the occupied $\mathrm{H}$ states are located over an energy interval starting from the valence band maximum and extending some 10 $\mathrm{eV}$ below. As regards to the unoccupied states, the $\mathrm{H}$ and $\mathrm{C}$ states are also extended and cover a wide energy range.

Being confident about the reliability of our ground state calculations, we now turn to the calculation of excited state properties. The GGA (full lines) and GW (dots) bandstructures for both conformers are presented in Fig 2 and 3 Firstly, our calculated GGA bandstructure for the chair conformation agrees well with the one of Sofo et al (notice that the bandstructure of the boat conformation was not reported in this paper). The minimum band gap is direct at the high symmetry point $\Gamma$ and has a value of $3.5 \mathrm{eV}$. However, this value is dramatically changed when using the more reliable GW approximation, in this case the value is $5.4 \mathrm{eV}$. The transitions at 


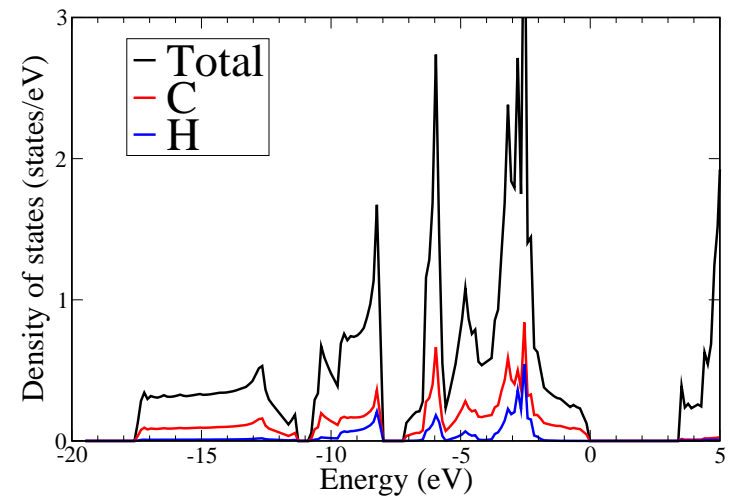

FIG. 1: (Color online) The total (in black) and partial (in red for carbon and in blue for hydrogen) density of states of graphane in the chair conformation. The top of the valence bands is chosen as the zero energy.

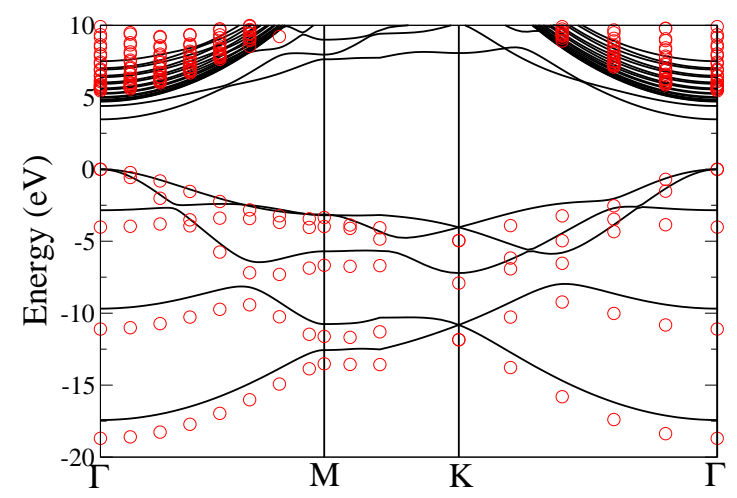

FIG. 2: (Color online) The GGA bandstructure (full lines) and the GW bandstructure (red dots) of graphane in the chair conformation. The top of the valence bands is chosen as the zero energy.

the high symmetry points $\mathrm{M}$ and $\mathrm{K}$ are also significantly increased, from $10.8 \mathrm{eV}$ and $12.2 \mathrm{eV}$ with the GGA approximation, to $13.7 \mathrm{eV}$ and $15.9 \mathrm{eV}$ with the $\mathrm{GW}$ approximation (see Table I). For the boat conformation (Fig. 3), the band gap is also found to be direct at the $\Gamma$ point, with a value given by the $\mathrm{GW}$ approximation of $4.9 \mathrm{eV}$, whereas the GGA gives only $3.3 \mathrm{eV}$. The values of the transitions at the $\mathrm{X}, \mathrm{S}$, and $\mathrm{Y}$ high-symmetry points are also notably corrected, see Table [1. Our calculations show that graphane, in both conformers, can be qualified as a large band gap insulator, and the electronic properties are expected to be quite different from the ones of graphene. This is qualitatively in agreement with observations.

Since graphane is a wide band-gap material it is relevant to ask if defect states can be introduced easily, and manipulated for electronics applications. We have here considered two examples of defects in graphane, in the chair conformation: replacement of a $\mathrm{H}$ atom by an

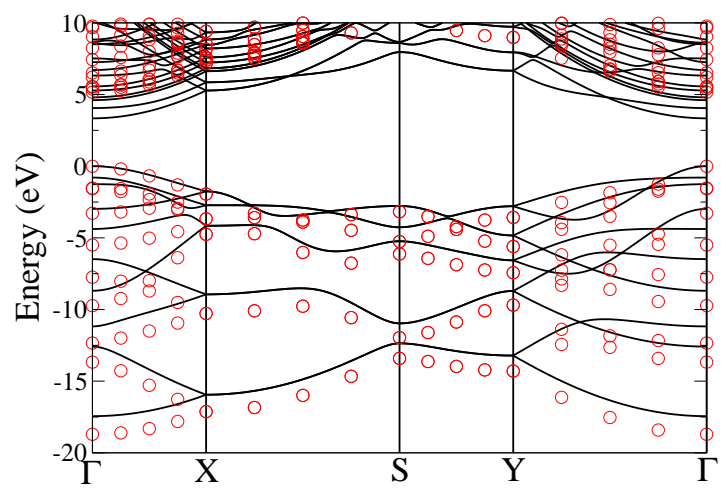

FIG. 3: (Color online) The GGA bandstructure (full lines) and the GW bandstructure (red dots) of graphane in the boat conformation. The top of the valence bands is chosen as the zero energy.

TABLE I: Values in $\mathrm{eV}$ of the transition energies of graphane at some high-symmetry points of the Brillouin zone for both conformers (chair or boat). The minimum band gap occurs at the $\Gamma$ point. The last two lines refer to calculations performed with a $2 \times 2$ supercell, in which either an hydroxyl group $(\mathrm{OH})$ or a $\mathrm{H}$ vacancy was introduced.

\begin{tabular}{|c|c|c|c|}
\hline Conformation & Transition & GGA value $(\mathrm{eV})$ & $\mathrm{GW}$ value $(\mathrm{eV})$ \\
\hline \hline Chair & $\Gamma_{v} \rightarrow \Gamma_{c}$ & 3.5 & 5.4 \\
\hline & $\mathrm{M}_{v} \rightarrow \mathrm{M}_{c}$ & 10.8 & 13.7 \\
\hline & $\mathrm{K}_{v} \rightarrow \mathrm{K}_{c}$ & 12.2 & 15.9 \\
\hline Boat & $\Gamma_{v} \rightarrow \Gamma_{c}$ & 3.3 & 5.1 \\
\hline & $\mathrm{X}_{v} \rightarrow \mathrm{X}_{c}$ & 7.0 & 9.0 \\
\hline & $\mathrm{S}_{v} \rightarrow \mathrm{S}_{c}$ & 10.7 & 13.9 \\
\hline & $\mathrm{Y}_{v} \rightarrow \mathrm{Y}_{c}$ & 9.4 & 12.6 \\
\hline Chair+ OH & $\Gamma_{v} \rightarrow \Gamma_{c}$ & 3.3 & 5.0 \\
\hline Chair+ H vacancy & $\Gamma_{v} \rightarrow \Gamma_{c}$ & 3.7 & 5.4 \\
\hline
\end{tabular}

hydroxyl group $(\mathrm{OH})$, and $\mathrm{H}$ vacancies. We have calculated the electronic structure of such systems using a $3 \times 3$ supercell, taking fully into account the relaxation of the structure due to the hydroxyl group or the $\mathrm{H}$ vacancy. For both cases, a $2 \times 2 \times 1$ kpoints mesh was used to obtain reliable geometry, and a final calculation with $8 \times 8 \times 1$ kpoints mesh was conducted to obtain well converged density of states.

In Fig. 4. we present our density of states for graphane $+\mathrm{OH}$. The partial density of states of oxygen is presented for 's' and 'p'states. It appears that the electronic structure of graphane is not significantly modified by the replacement of a $\mathrm{H}$ atom by the hydroxyl group. While the O-s states are very widely distributed in energy, the O-p states appear mainly as a peak close to the maximum of the valence band, between -1 and $-2 \mathrm{eV}$. Among the extra electrons brought by the oxy- 


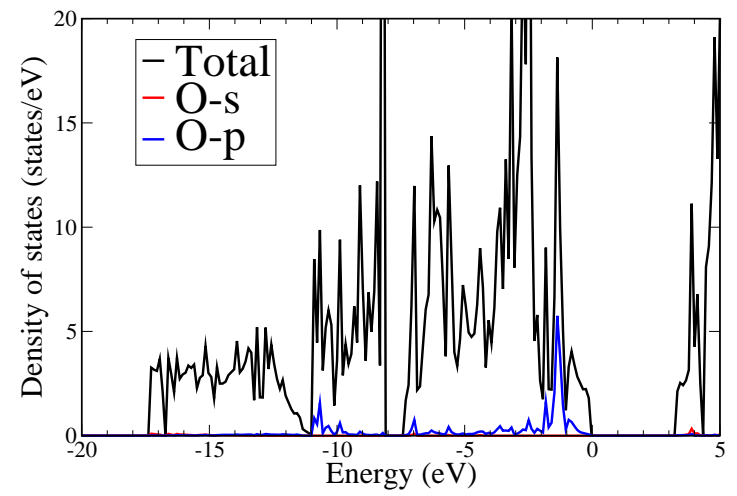

FIG. 4: (Color online) The total (in black) and partial (in red for O-s states and in blue for O-p states) density of states of graphane+OH. The top of the valence bands is chosen as the zero energy.

gen atom, one electron is involved in a bond with the neighbouring $\mathrm{C}$ atom, another electron is participating in the bond with $\mathrm{H}$, and the two remaining electrons are forming a doublet on the oxygen atom. This is confirmed by the fact that the geometry of the network of carbon atoms, driven by $\mathrm{sp}^{3}$ hybridization, is almost unaffected by the addition of the hydroxyl group. The band gap (from GGA) is slightly decreased, from $3.5 \mathrm{eV}$ for pure graphane, to $3.3 \mathrm{eV}$ here. Here as well, the band gap is underestimated by GGA, so we have applied the GW approximation to get a meaningful value. However, since the numerical cost of the GW approximation is quite high, we had to reduce the size of the supercell to $2 \times 2$.

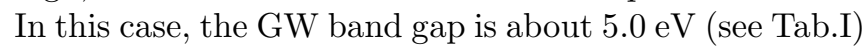
which is in line with the band gap of pure graphane $(5.4$ $\mathrm{eV})$.

Next we introduced a hydrogen vacancy in graphane. Then, not every $\mathrm{C}$ atom of the graphane layer of the graphane layer is saturated with a $\mathrm{H}$ atom. From inspection of the relaxed geometry, it appears that the bonds between the carbon neighbouring the $\mathrm{H}$ vacancy and the carbon network have gained a significant part of $\mathrm{sp}^{2}$ hybridization, like in graphene, with bond lengths here of $1.49 \AA$ (1.42 $\AA$ in graphene). The resulting total density of states is shown in Fig 5 . An impurity state develops

close to $2 \mathrm{eV}$ above the valence band maximum, at the Fermi level, changing significantly the overall picture of the DOS. Also, using the GW approximation (for a $2 \times 2$ supercell), the difference in energy between the valence band maximum and the conduction band mininum is increased from $3.7 \mathrm{eV}$ to $5.4 \mathrm{eV}$, but the impurity state at the Fermi level is not affected by the GW correction.

To summarize, in this article, results concerning the electronic structure of graphane have been presented using the GW approximation. It was found that for both conformers, the minimim band gap is direct and located

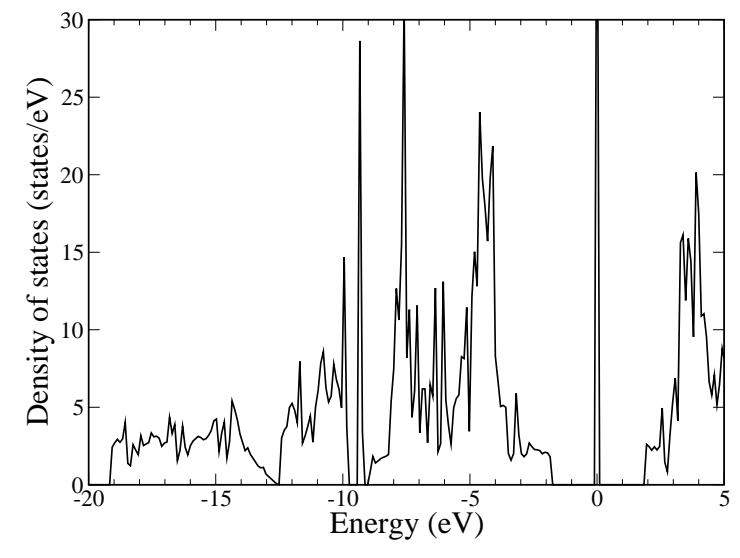

FIG. 5: The total density of states of graphane+ a $\mathrm{H}$ vacancy. The Fermi level is put at zero energy.

at the $\Gamma$ point. For the stable chair conformation, the most stable one, it has a value of $5.4 \mathrm{eV}$, while for the boat conformation, it has a value of $4.9 \mathrm{eV}$. We also found that defects in graphane, in the form of $\mathrm{H}$ vacancies, provide an impurity state some $2 \mathrm{eV}$ above the valence band maximum, a finding which may be important when trying to use this material for electronics applications.

S. L. is grateful to G. Kresse for providing the version 5 of VASP and acknowledges financial support from ANR PNANO Grant ANR- 06-NANO-053-02 and ANR Grant ANR-BLAN07-1-186138. M. K. and O.E. acknowledge financial support from Vetenskapsrådet (VR), Göran Gustafsson Stiftelse, and SNIC/SNAC. M. I. K. acknowledges financial support from FOM (Netherlands).

[1] K. S. Novoselov et al, Science 306, 666 (2004).

[2] A. K. Geim and K. S. Novoselov, Nature Mater. 6, 183 (2007).

[3] M. I. Katsnelson, Mater. Today 10, 20 (2007).

[4] A. H. Castro Neto, F. Guinea, N. M. R. Peres, K. S. Novoselov, and A. K. Geim, Rev. Mod. Phys. 81, 109 (2009).

[5] M. I. Katsnelson, K. S. Novoselov, and A. K. Geim, Nature Phys. 2, 620 (2006).

[6] L. A. Ponomarenko et al, Science 320, 356 (2008).

[7] D. C. Elias et al. Science 323, 610 (2009).

[8] J. O. Sofo, A. S. Chaudhari, and G. D. Barber, Phys. Rev. B 75, 153401 (2007).

[9] D. W. Boukhvalov, M. I. Katsnelson, and A. I. Lichtenstein, Phys. Rev. B 77, 035427 (2008).

[10] D. W. Boukhvalov and M. I. Katsnelson, arXiv:0809.5257 (to appear in J. Phys.: Condens. Matter).

[11] R. W. Godby, M. Schluter, and L. J. Sham, Phys. Rev. Lett 56, 2415 (1986).

[12] O.Wessely, M.I.Katsnelson, A.Nilsson, A.Nikitin, H.Ogasawara, B.Sanyal and O.Eriksson, Phys. Rev. B 76, 161402 (2007).

[13] P. Hohenberg and W. Kohn, Phys. Rev. 136 (1964); W. 
Kohn and L.J Sham, Phys. Rev. 140, A1113 (1965).

[14] L. Hedin, Phys. Rev. 139, A796 (1965).

[15] L. Hedin and S. Lundquist, in Solid State Physics, edited by H. Ehrenreich, F. Seitz, and D. Turnbull (Academic, New York, 1969), Vol. 23, p. 1.

[16] F. Aryasetiawan and O. Gunnarsson, Rep. Prog. Phys. 61, 237-312 (1998).

[17] W. G. Aulbur, L. Jönsson, and J. W. Wilkins, 'Quasiparticle calculations in solids', in Solid State Physics; edited by H. Ehrenreich and F. Spaegen, vol $\mathbf{5 4 .}$

[18] G. Onida, L. Reining, and A. Rubio, Rev. Mod. Phys. 74, 601 (2002).

[19] G. Kresse and D. Joubert, Phys. Rev. B. 59, 1758 (1999).

[20] P.E Blöchl, Phys. Rev. B 50, 17953 (1994).

[21] J. P. Perdew, K. Burke, and M. Ernzerhof, Phys. Rev. Lett. 77, 3865 (1996).
[22] H. J. Monkhorst and J.D. Pack, Phys. Rev. B 13, 5188 (1976).

[23] M. Shishkin and G. Kresse Phys. Rev. B 74, 035101 (2006).

[24] B. Arnaud and M. Alouani Phys. Rev. B 62, 4464 (2000).

[25] S. Lebègue, B. Arnaud, M. Alouani, and P. E. Blöchl Phys. Rev. B 67, 155208 (2003).

[26] Y-W Son M. L. Cohen and S. G. Louie Phys. Rev. Lett. 97, 216803 (2006)

[27] L Yang, C-H Park, Y-W Son, M. L. Cohen, and S. G. Louie Phys. Rev. Lett. 99, 186801 (2007)

[28] P. E. Trevisanutto,C. Giorgetti, L. Reining, M. Ladisa and V. Olevano Phys. Rev. Lett. 101, 226405 (2008)

[29] T. Miyake and S. Saita Phys. Rev. B 68, 155424 (2003) 